Research Article

\title{
General Iterative Methods for Equilibrium Problems and Infinitely Many Strict Pseudocontractions in Hilbert Spaces
}

\author{
Peichao Duan and Aihong Wang \\ College of Science, Civil Aviation University of China, Tianjin 300300, China \\ Correspondence should be addressed to Peichao Duan, pcduancauc@126.com \\ Received 11 January 2012; Revised 24 February 2012; Accepted 25 February 2012 \\ Academic Editor: Rudong Chen
}

Copyright (C) 2012 P. Duan and A. Wang. This is an open access article distributed under the Creative Commons Attribution License, which permits unrestricted use, distribution, and reproduction in any medium, provided the original work is properly cited.

We propose an implicit iterative scheme and an explicit iterative scheme for finding a common element of the set of fixed point of infinitely many strict pseudocontractive mappings and the set of solutions of an equilibrium problem by the general iterative methods. In the setting of real Hilbert spaces, strong convergence theorems are proved. Our results improve and extend the corresponding results reported by many others.

\section{Introduction}

Let $H$ be a real Hilbert space and let $C$ be a nonempty closed convex subset of $H$. Let $F$ be a bifunction from $C \times C$ to $\mathbb{R}$, where $\mathbb{R}$ is the set of real numbers.

The equilibrium problem for $F: C \times C \rightarrow \mathbb{R}$ is to find $x \in C$ such that

$$
F(x, y) \geq 0
$$

for all $y \in C$. The set of such solutions is denoted by $\operatorname{EP}(F)$.

A mapping $S$ of $C$ is said to be a $\mathcal{K}$-strict pseudocontraction if there exists a constant $\kappa \in[0,1)$ such that

$$
\|S x-S y\|^{2} \leq\|x-y\|^{2}+\kappa\|(I-S) x-(I-S) y\|^{2}
$$

for all $x, y \in C$; see [1]. We denote the set of fixed points of $S$ by $F(S)$ (i.e., $F(S)=\{x \in C$ : $S x=x\}$ ). 
Note that the class of strict pseudocontractions strictly includes the class of nonexpansive mappings which are mapping $S$ on $C$ such that

$$
\|S x-S y\| \leq\|x-y\|
$$

for all $x, y \in C$. That is, $S$ is nonexpansive if and only if $S$ is a 0 -strict pseudocontraction.

Numerous problems in physics, optimization, and economics reduce to finding a solution of the equilibrium problem. Some methods have been proposed to solve the equilibrium problem (1.1); see, for instance, [2-4]. In particular, Combettes and Hirstoaga [5] proposed several methods for solving the equilibrium problem. On the other hand, Mann [6], Shimoji and Takahashi [7] considered iterative schemes for finding a fixed point of a nonexpansive mapping. Further, Acedo and $\mathrm{Xu}[8]$ projected new iterative methods for finding a fixed point of strict pseudocontractions.

In 2006, Marino and Xu [3] introduced the general iterative method and proved that the algorithm converged strongly. Recently, Liu [2] considered a general iterative method for equilibrium problems and strict pseudocontractions. Tian [9] proposed a new general iterative algorithm combining an $L$-Lipschitzian and $\eta$-strong monotone operator. Very recently, Wang [10] considered a general composite iterative method for infinite family strict pseudocontractions.

In this paper, motivated by the above facts, we introduce two iterative schemes and obtain strong convergence theorems for finding a common element of the set of fixed points of a infinite family of strict pseudocontractions and the set of solutions of the equilibrium problem (1.1).

\section{Preliminaries}

Throughout this paper, we always write $\rightarrow$ for weak convergence and $\rightarrow$ for strong convergence. We need some facts and tools in a real Hilbert space $H$ which are listed as below.

Lemma 2.1. Let $H$ be a real Hilbert space. There hold the following identities:

(i) $\|x-y\|^{2}=\|x\|^{2}-\|y\|^{2}-2\langle x-y, y\rangle, \forall x, y \in H$;

(ii) $\|t x+(1-t) y\|^{2}=t\|x\|^{2}+(1-t)\|y\|^{2}-t(1-t)\|x-y\|^{2}, \forall t \in[0,1], \forall x, y \in H$.

Lemma 2.2 (see [11]). Assume that $\left\{\alpha_{n}\right\}$ is a sequence of nonnegative real numbers such that

$$
\alpha_{n+1} \leq\left(1-\gamma_{n}\right) \alpha_{n}+\delta_{n}
$$

where $\left\{\gamma_{n}\right\}$ is a sequence in $(0,1)$ and $\left\{\delta_{n}\right\}$ is a sequence such that

(i) $\sum_{n=1}^{\infty} \gamma_{n}=\infty$;

(ii) $\lim _{n \rightarrow \infty} \sup \left(\delta_{n} / \gamma_{n}\right) \leq 0$ or $\sum_{n=1}^{\infty}\left|\delta_{n}\right|<\infty$.

Then, $\lim _{n \rightarrow \infty} \alpha_{n}=0$. 
Recall that given a nonempty closed convex subset $C$ of a real Hilbert space $H$, for any $x \in H$, there exists a unique nearest point in $C$, denoted by $P_{C} x$, such that

$$
\left\|x-P_{C} x\right\| \leq\|x-y\|
$$

for all $y \in C$. Such a $P_{C}$ is called the metric (or the nearest point) projection of $H$ onto $C$. As known, $y=P_{C} x$ if and only if there holds the relation:

$$
\langle x-y, y-z\rangle \geq 0 \quad \forall z \in C
$$

Lemma 2.3 (see [10]). Let $A: H \rightarrow H$ be a L-Lipschitzian and $\eta$-strongly monotone operator on a Hilbert space $H$ with $L>0, \eta>0,0<\mu<2 \eta / L^{2}$, and $0<t<1$. Then, $S=(I-t \mu A): H \rightarrow H$ is a contraction with contractive coefficient $1-t \tau$ and $\tau=(1 / 2) \mu\left(2 \eta-\mu L^{2}\right)$.

Lemma 2.4 (see [1]). Let $S: C \rightarrow C$ be a $\kappa$-strict pseudocontraction. Define $T: C \rightarrow C$ by $T x=\lambda x+(1-\lambda) S x$ for each $x \in C$. Then, as $\lambda \in[\kappa, 1), T$ is a nonexpansive mapping such that $F(T)=F(S)$.

Lemma 2.5 (see [9]). Let $H$ be a Hilbert space and $f: H \rightarrow H$ be a contraction with coefficient $0<\alpha<1$, and $A: H \rightarrow H$ an L-Lipschitzian continuous operator and $\eta$-strongly monotone with $L>0, \eta>0$. Then for $0<\gamma<\mu \eta / \alpha$ :

$$
\langle x-y,(\mu A-\gamma f) x-(\mu A-\gamma f) y\rangle \geq(\mu \eta-\gamma \alpha)\|x-y\|^{2}, \quad x, y \in H .
$$

That is, $\mu A-\gamma f$ is strongly monotone with coefficient $\mu \eta-\gamma \alpha$.

Let $\left\{S_{n}\right\}$ be a sequence of $\kappa_{n}$-strict pseudo-contractions. Define $S_{n}^{\prime}=\theta_{n} I+\left(1-\theta_{n}\right) S_{n}, \theta_{n} \in$ $\left[\kappa_{n}, 1\right)$. Then, by Lemma $2.4, S_{n}^{\prime}$ is nonexpansive. In this paper, consider the mapping $W_{n}$ defined by

$$
\begin{aligned}
& U_{n, n+1}=I, \\
& U_{n, n}=t_{n} S_{n}^{\prime} U_{n, n+1}+\left(1-t_{n}\right) I \\
& U_{n, n-1}=t_{n-1} S_{n-1}^{\prime} U_{n, n}+\left(1-t_{n-1}\right) I \\
& \ldots, \\
& U_{n, i}=t_{i} S_{i}^{\prime} U_{n, i+1}+\left(1-t_{i}\right) I \\
& \ldots, \\
& U_{n, 2}=t_{2} S_{2}^{\prime} U_{n, 3}+\left(1-t_{2}\right) I \\
& W_{n}=U_{n, 1}=t_{1} S_{1}^{\prime} U_{n, 2}+\left(1-t_{1}\right) I
\end{aligned}
$$

where $t_{1}, t_{2}, \ldots$ are real numbers such that $0 \leq t_{n}<1$. Such a mapping $W_{n}$ is called a $W$-mapping generated by $S_{1}^{\prime}, S_{2}^{\prime}, \ldots$ and $t_{1}, t_{2}, \ldots$ It is easy to see $W_{n}$ is nonexpansive.

Lemma 2.6 (see [7]). Let $C$ be a nonempty closed convex subset of a strictly convex Banach space $E$, let $S_{1}^{\prime}, S_{2}^{\prime}, \ldots$ be nonexpansive mappings of $C$ into itself such that $\cap_{i=1}^{\infty} F\left(S_{i}^{\prime}\right) \neq \emptyset$ and let $t_{1}, t_{2}, \ldots$ be 
real numbers such that $0<t_{i} \leq b<1$, for every $i=1,2, \ldots$ Then, for any $x \in C$ and $k \in N$, the limit $\lim _{n \rightarrow \infty} U_{n, k} x$ exists.

Using Lemma 2.6, one can define the mapping $W$ of $C$ into itself as follows:

$$
W x:=\lim _{n \rightarrow \infty} W_{n} x=\lim _{n \rightarrow \infty} U_{n, 1} x, \quad x \in C .
$$

Lemma 2.7 (see [7]). Let $C$ be a nonempty closed convex subset of a strictly convex Banach space $E$. Let $S_{1}^{\prime}, S_{2}^{\prime}, \ldots$ be nonexpansive mappings of $C$ into itself such that $\cap_{i=1}^{\infty} F\left(S_{i}^{\prime}\right) \neq \emptyset$ and let $t_{1}, t_{2}, \ldots$ be real numbers such that $0<t_{i} \leq b<1$, for all $i \geq 1$. If $K$ is any bounded subset of $C$, then

$$
\lim _{n \rightarrow \infty} \sup _{x \in K}\left\|W x-W_{n} x\right\|=0
$$

Lemma 2.8 (see [12]). Let $C$ be a nonempty closed convex subset of a Hilbert space $H$, let $\left\{S_{i}^{\prime}: C \rightarrow\right.$ C) be a family of infinite nonexpansive mappings with $\cap_{i=1}^{\infty} F\left(S_{i}^{\prime}\right) \neq \emptyset$, let $t_{1}, t_{2}, \ldots$ be real numbers such that $0<t_{i} \leq b<1$, for every $i=1,2, \ldots$. Then $F(W)=\cap_{i=1}^{\infty} F\left(S_{i}^{\prime}\right)$. conditions:

For solving the equilibrium problem, assume that the bifunction $F$ satisfies the following

(A1) $F(x, x)=0$ for all $x \in C$;

(A2) $F$ is monotone, that is, $F(x, y)+F(y, x) \leq 0$ for any $x, y \in C$;

(A3) for each $x, y, z \in C, \limsup _{t \rightarrow 0} F(t z+(1-t) x, y) \leq F(x, y)$;

(A4) $F(x, \cdot)$ is convex and lower semicontinuous for each $x \in C$.

Recall some lemmas which will be needed in the rest of this paper.

Lemma 2.9 (see [13]). Let $C$ be a nonempty closed convex subset of $H$, let $F$ be bifunction from $C \times C$ to $\mathbb{R}$ satisfying (A1)-(A4), and let $r>0$ and $x \in H$. Then, there exists $z \in C$ such that

$$
F(z, y)+\frac{1}{r}\langle y-z, z-x\rangle \geq 0, \quad \forall y \in C
$$

Lemma 2.10 (see [5]). For $r>0, x \in H$, define a mapping $T_{r}: H \rightarrow C$ as follows:

$$
T_{r}(x)=\left\{z \in C \mid F(z, y)+\frac{1}{r}\langle y-z, z-x\rangle \geq 0, \forall y \in C\right\}
$$

for all $x \in H$. Then, the following statements hold:

(i) $T_{r}$ is single-valued;

(ii) $T_{r}$ is firmly nonexpansive, that is, for any $x, y \in H$,

$$
\left\|T_{r} x-T_{r} y\right\|^{2} \leq\left\langle T_{r} x-T_{r} y, x-y\right\rangle
$$

(iii) $F\left(T_{r}\right)=E P(F)$;

(iv) $E P(F)$ is closed and convex. 
Lemma 2.11 (see [14]). Let $\left\{x_{n}\right\}$ and $\left\{z_{n}\right\}$ be bounded sequences in a Banach space and let $\left\{\beta_{n}\right\}$ be a sequence of real numbers such that $0<\liminf _{n \rightarrow \infty} \beta_{n} \leq \limsup _{n \rightarrow \infty} \beta_{n}<1$ for all $n=0,1,2, \ldots$. Suppose that $x_{n+1}=\left(1-\beta_{n}\right) z_{n}+\beta_{n} x_{n}$ for all $n=0,1,2, \ldots$ and $\limsup _{n \rightarrow \infty}\left\|z_{n+1}-z_{n}\right\|-\left\|x_{n+1}-x_{n}\right\| \leq$ 0. Then $\lim _{n \rightarrow \infty}\left\|z_{n}-x_{n}\right\|=0$.

Lemma 2.12 (see [4]). Let $C, H, F$, and $T_{r} x$ be as in Lemma 2.10. Then, the following holds:

$$
\left\|T_{s} x-T_{t} x\right\|^{2} \leq \frac{s-t}{s}\left\langle T_{s} x-T_{t} x, T_{s} x-x\right\rangle
$$

for all $s, t>0$ and $x \in H$.

Lemma 2.13 (see [10]). Let $H$ be a Hilbert space and let $C$ be a nonempty closed convex subset of $H$, and $T: C \rightarrow C$ a nonexpansive mapping with $F(T) \neq \emptyset$. If $\left\{x_{n}\right\}$ is a sequence in $C$ weakly converging to $x$ and if $\left\{(I-T) x_{n}\right\}$ converges strongly to $y$, then $(I-T) x=y$.

\section{Main Result}

Throughout the rest of this paper, we always assume that $f$ is a contraction of $H$ into itself with coefficient $\alpha \in(0,1)$, and $A$ is a $L$-Lipschitzian continuous operator and $\eta$-strongly monotone on $H$ with $L>0, \eta>0$. Assume that $0<\mu<2 \eta / L^{2}$ and $0<\gamma<\mu\left(\eta-\left(\mu L^{2} / 2\right)\right) / \alpha=$ $\tau / \alpha$.

Define a mapping $V_{n}=\beta_{n} I+\left(1-\beta_{n}\right) W_{n} T_{r_{n}}$. Since both $W_{n}$ and $T_{r_{n}}$ are nonexpansive, it is easy to get $V_{n}$ is also nonexpansive. Consider the following mapping $G_{n}$ on $H$ defined by

$$
G_{n} x=\alpha_{n} \gamma f(x)+\left(I-\alpha_{n} \mu A\right) V_{n} x, \quad \forall x \in H, n \in N,
$$

where $\alpha_{n} \in(0,1)$. By Lemmas 2.3 and 2.10, we have

$$
\begin{aligned}
\left\|G_{n} x-G_{n} y\right\| & \leq \alpha_{n} \gamma\|f(x)-f(y)\|+\left(1-\alpha_{n} \tau\right)\left\|V_{n} x-V_{n} y\right\| \\
& \leq \alpha_{n} \gamma \alpha\|x-y\|+\left(1-\alpha_{n} \tau\right)\|x-y\| \\
& =\left(1-\alpha_{n}(\tau-\gamma \alpha)\right)\|x-y\| .
\end{aligned}
$$

Since $0<1-\alpha_{n}(\tau-\gamma \alpha)<1$, it follows that $G_{n}$ is a contraction. Therefore, by the Banach contraction principle, $G_{n}$ has a unique fixed pointed $x_{n}^{f} \in H$ such that

$$
x_{n}^{f}=\alpha_{n} \gamma f\left(x_{n}^{f}\right)+\left(I-\alpha_{n} \mu A\right) V_{n} x_{n}^{f} .
$$

For simplicity, we will write $x_{n}$ for $x_{n}^{f}$ provided no confusion occurs. Next we prove the sequences $\left\{x_{n}\right\}$ converges strongly to a $x^{*} \in \Omega=\cap_{i=1}^{\infty} F\left(S_{i}\right) \cap E P(F)$ which solves the variational inequality:

$$
\left\langle(\gamma f-\mu A) x^{*}, p-x^{*}\right\rangle \leq 0, \quad \forall p \in \Omega
$$

Equivalently, $x^{*}=P_{\Omega}(I-\mu A+\gamma f) x^{*}$. 
Theorem 3.1. Let $C$ be a nonempty closed convex subset of a real Hilbert space $H$ and $F$ a bifunction from $C \times C$ to $\mathbb{R}$ satisfying (A1)-(A4). Let $S_{i}: C \rightarrow C$ be a family $\kappa_{i}$-strict pseudocontractions for some $0 \leq \kappa_{i}<1$. Assume the set $\Omega=\cap_{i=1}^{\infty} F\left(S_{i}\right) \cap E P(F) \neq \emptyset$. Let $f$ be a contraction of $H$ into itself with $\alpha \in(0,1)$ and let $A$ be a L-Lipschitzian continuous operator and $\eta$-strongly monotone with $L>0, \eta>0,0<\mu<2 \eta / L^{2}$ and $0<\gamma<\mu\left(\eta-\left(\mu L^{2} / 2\right)\right) / \alpha=\tau / \alpha$. For every $n \in \mathbb{N}$, let $W_{n}$ be the mapping generated by $S_{i}^{\prime}$ and $t_{i}$ as in (2.5). Let $\left\{x_{n}\right\}$ and $\left\{u_{n}\right\}$ be sequences generated by the following algorithm:

$$
\begin{gathered}
u_{n}=T_{r_{n}} x_{n}, \\
y_{n}=\beta_{n} x_{n}+\left(1-\beta_{n}\right) W_{n} u_{n}, \\
x_{n}=\alpha_{n} \gamma f\left(x_{n}\right)+\left(I-\mu \alpha_{n} A\right) y_{n} .
\end{gathered}
$$

If $\left\{\alpha_{n}\right\},\left\{\beta_{n}\right\}$, and $\left\{r_{n}\right\}$ satisfy the following conditions:

(i) $\left\{\alpha_{n}\right\} \subset(0,1), \lim _{n \rightarrow \infty} \alpha_{n}=0$;

(ii) $0<\liminf _{n \rightarrow \infty} \beta_{n} \leq \lim \sup _{n \rightarrow \infty} \beta_{n}<1$;

(iii) $\left\{r_{n}\right\} \subset(0, \infty), \liminf _{n \rightarrow \infty} r_{n}>0$.

Then, $\left\{x_{n}\right\}$ converges strongly to a point $x^{*} \in \Omega$, which solves the variational inequality (3.4).

Proof. The proof is divided into several steps.

Step 1. Show first that $\left\{x_{n}\right\}$ is bounded.

Take any $p \in \Omega$, by (3.5) and Lemma 2.3, we derive that

$$
\begin{aligned}
\left\|x_{n}-p\right\| & =\left\|\alpha_{n}\left(\gamma f\left(x_{n}\right)-\mu A p\right)+\left(I-\mu \alpha_{n} A\right) y_{n}-\left(I-\mu \alpha_{n} A\right) p\right\| \\
& \leq \alpha_{n} \alpha \gamma\left\|x_{n}-p\right\|+\alpha_{n}\|\gamma f(p)-\mu A p\|+\left(1-\alpha_{n} \tau\right)\left\|y_{n}-p\right\| \\
& \leq\left(1-\alpha_{n}(\tau-\gamma \alpha)\right)\left\|x_{n}-p\right\|+\alpha_{n}\|\gamma f(p)-\mu A p\| .
\end{aligned}
$$

It follows that $\left\|x_{n}-p\right\| \leq(\|\gamma f(p)-\mu A p\|) /(\tau-\gamma \alpha)$.

Hence, $\left\{x_{n}\right\}$ is bounded, so are $\left\{u_{n}\right\}$ and $\left\{y_{n}\right\}$. It follows from the Lipschitz continuity of $A$ that $\left\{A x_{n}\right\}$ and $\left\{A u_{n}\right\}$ are also bounded. From the nonexpansivity of $f$ and $W_{n}$, it follows that $\left\{f\left(x_{n}\right)\right\}$ and $\left\{W_{n} x_{n}\right\}$ are also bounded.

Step 2. Show that

$$
\lim _{n \rightarrow \infty}\left\|u_{n}-x_{n}\right\|=0, \quad \lim _{n \rightarrow \infty}\left\|u_{n}-y_{n}\right\|=0
$$

Notice that

$$
\left\|u_{n}-y_{n}\right\| \leq\left\|u_{n}-x_{n}\right\|+\left\|x_{n}-y_{n}\right\|=\left\|u_{n}-x_{n}\right\|+\alpha_{n}\left\|\gamma f\left(x_{n}\right)-\mu A y_{n}\right\|
$$


Journal of Applied Mathematics

By Lemma 2.10, we have

$$
\left\|u_{n}-p\right\|^{2}=\left\|T_{r_{n}} x_{n}-T_{r_{n}} p\right\|^{2} \leq\left\langle x_{n}-p, u_{n}-p\right\rangle=\frac{1}{2}\left(\left\|u_{n}-p\right\|^{2}+\left\|x_{n}-p\right\|^{2}-\left\|x_{n}-u_{n}\right\|^{2}\right) .
$$

It follows that

$$
\left\|u_{n}-p\right\|^{2} \leq\left\|x_{n}-p\right\|^{2}-\left\|x_{n}-u_{n}\right\|^{2}
$$

Thus, from Lemma 2.1 and (3.10), we get

$$
\begin{aligned}
\left\|x_{n}-p\right\|^{2} \\
\quad=\left\|\alpha_{n}\left(\gamma f\left(x_{n}\right)-\mu A p\right)+\left(I-\mu \alpha_{n} A\right) y_{n}-\left(I-\mu \alpha_{n} A\right) p\right\|^{2} \\
\quad \leq\left(1-\alpha_{n} \tau\right)^{2}\left\|y_{n}-p\right\|^{2}+2 \alpha_{n}\left\langle\gamma f\left(x_{n}\right)-\gamma f(p)+\gamma f(p)-\mu A p, x_{n}-p\right\rangle \\
\quad \leq\left(1-\alpha_{n} \tau\right)^{2}\left\|u_{n}-p\right\|^{2}+2 \alpha_{n}\left\langle\gamma f\left(x_{n}\right)-\gamma f(p)+\gamma f(p)-\mu A p, x_{n}-p\right\rangle \\
\quad \leq\left(1-\alpha_{n} \tau\right)^{2}\left(\left\|x_{n}-p\right\|^{2}-\left\|x_{n}-u_{n}\right\|^{2}\right)+2 \alpha_{n} \gamma \alpha\left\|x_{n}-p\right\|^{2}+2 \alpha_{n}\|\gamma f(p)-\mu A p\|\left\|x_{n}-p\right\| \\
\quad=\left(1-2 \alpha_{n}(\tau-\gamma \alpha)+\left(\alpha_{n} \tau\right)^{2}\right)\left\|x_{n}-p\right\|^{2}-\left(1-\alpha_{n} \tau\right)^{2}\left\|x_{n}-u_{n}\right\|^{2}+2 \alpha_{n}\|\gamma f(p)-\mu A p\|\left\|x_{n}-p\right\| \\
\quad \leq\left\|x_{n}-p\right\|^{2}+\left(\alpha_{n} \tau\right)^{2}\left\|x_{n}-p\right\|^{2}-\left(1-\alpha_{n} \tau\right)^{2}\left\|x_{n}-u_{n}\right\|^{2}+2 \alpha_{n}\|\gamma f(p)-\mu A p\|\left\|x_{n}-p\right\| .
\end{aligned}
$$

It follows that

$$
\left(1-\alpha_{n} \tau\right)^{2}\left\|x_{n}-u_{n}\right\|^{2} \leq\left(\alpha_{n} \tau\right)^{2}\left\|x_{n}-p\right\|^{2}+2 \alpha_{n}\|\gamma f(p)-\mu A p\|\left\|x_{n}-p\right\| .
$$

Since $\alpha_{n} \rightarrow 0$, we have

$$
\lim _{n \rightarrow \infty}\left\|u_{n}-x_{n}\right\|=0
$$

From (3.8), it is easy to get

$$
\lim _{n \rightarrow \infty}\left\|u_{n}-y_{n}\right\|=0
$$

Step 3. Show that

$$
\begin{gathered}
\lim _{n \rightarrow \infty}\left\|u_{n}-W u_{n}\right\|=0 \\
\left\|u_{n}-W_{n} u_{n}\right\| \leq\left\|u_{n}-y_{n}\right\|+\left\|y_{n}-W_{n} u_{n}\right\|=\left\|u_{n}-y_{n}\right\|+\beta_{n}\left(\left\|x_{n}-u_{n}\right\|+\left\|u_{n}-W_{n} u_{n}\right\|\right) .
\end{gathered}
$$


This implies that

$$
\left(1-\beta_{n}\right)\left\|u_{n}-W_{n} u_{n}\right\| \leq\left\|u_{n}-y_{n}\right\|+\beta_{n}\left\|x_{n}-u_{n}\right\| .
$$

From condition (ii), (3.13), and (3.14), we have

$$
\left\|u_{n}-W_{n} u_{n}\right\| \longrightarrow 0
$$

Notice that

$$
\left\|u_{n}-W u_{n}\right\| \leq\left\|u_{n}-W_{n} u_{n}\right\|+\left\|W_{n} u_{n}-W u_{n}\right\|
$$

By Lemma 2.7 and (3.18), we get (3.15).

Since $\left\{u_{n}\right\}$ is bounded, so there exists a subsequence $\left\{u_{n_{j}}\right\}$ which converges weakly to $x^{*}$.

Step 4. Show that $x^{*} \in \Omega$.

Since $C$ is closed and convex, $C$ is weakly closed. So, we have $x^{*} \in C$.

From (3.15), we obtain $W u_{n_{j}} \rightarrow x^{*}$. From Lemmas 2.8, 2.4, and 2.13, we have $x^{*} \in$ $F(W)=\cap_{i=1}^{\infty} F\left(S_{i}^{\prime}\right)=\cap_{i=1}^{\infty} F\left(S_{i}\right)$.

By $u_{n}=T_{r_{n}} x_{n}$, for all $n \geq 1$, we have

$$
F\left(u_{n}, y\right)+\frac{1}{r_{n}}\left\langle y-u_{n}, u_{n}-x_{n}\right\rangle \geq 0, \quad \forall y \in C
$$

It follows from (A2) that

$$
\frac{1}{r_{n}}\left\langle y-u_{n}, u_{n}-x_{n}\right\rangle \geq F\left(y, u_{n}\right), \quad \forall y \in C
$$

Hence, we get

$$
\frac{1}{r_{n_{j}}}\left\langle y-u_{n_{j}}, u_{n_{j}}-x_{n_{j}}\right\rangle \geq F\left(y, u_{n_{j}}\right), \quad \forall y \in C
$$

It follows from condition (iii), (3.13), and (A4) that

$$
0 \geq F\left(y, x^{*}\right), \quad \forall \mathrm{y} \in C
$$

For $s$ with $0<s \leq 1$ and $y \in C$, let $y_{s}=s y+(1-s) x^{*}$. Since $y \in C$ and $x^{*} \in C$, we obtain $y_{s} \in C$ and hence $F\left(y_{s}, x^{*}\right) \leq 0$. So, we have

$$
0=f\left(y_{s}, y_{s}\right) \leq s F\left(y_{s}, y\right)+(1-s) F\left(y_{s}, x^{*}\right) \leq s F\left(y_{s}, y\right)
$$


Dividing by $s$, we get

$$
F\left(y_{s}, \mathrm{y}\right) \geq 0, \quad \forall y \in C
$$

Letting $s \rightarrow 0$ and from (A3), we get

$$
F\left(x^{*}, y\right) \geq 0
$$

for all $y \in C$ and $x^{*} \in \operatorname{EP}(F)$. Hence $x^{*} \in \Omega$.

Step 5. Show that $x_{n} \rightarrow x^{*}$, where $x^{*}=P_{\Omega}(I-\mu A+\gamma f) x^{*}$ :

$$
x_{n}-x^{*}=\alpha_{n}\left(\gamma f\left(x_{n}\right)-\mu A x^{*}\right)+\left(I-\mu \alpha_{n} A\right) y_{n}-\left(I-\mu \alpha_{n} A\right) x^{*} .
$$

Hence, we obtain

$$
\begin{aligned}
\left\|x_{n}-x^{*}\right\|^{2} & =\alpha_{n}\left\langle\gamma f\left(x_{n}\right)-\mu A x^{*}, x_{n}-x^{*}\right\rangle+\left\langle\left(I-\mu \alpha_{n} A\right) y_{n}-\left(I-\mu \alpha_{n} A\right) x^{*}, x_{n}-x^{*}\right\rangle \\
& \leq \alpha_{n}\left\langle\gamma f\left(x_{n}\right)-\mu A x^{*}, x_{n}-x^{*}\right\rangle+\left(1-\alpha_{n} \tau\right)\left\|x_{n}-x^{*}\right\|^{2} .
\end{aligned}
$$

It follows that

$$
\begin{aligned}
\left\|x_{n}-x^{*}\right\|^{2} & \leq \frac{1}{\tau}\left\langle\gamma f\left(x_{n}\right)-\mu A x^{*}, x_{n}-x^{*}\right\rangle \\
& =\frac{1}{\tau}\left(\gamma\left\langle f\left(x_{n}\right)-f\left(x^{*}\right), x_{n}-x^{*}\right\rangle+\left\langle\gamma f\left(x^{*}\right)-\mu A x^{*}, x_{n}-x^{*}\right\rangle\right) \\
& \leq \frac{1}{\tau}\left(\gamma \alpha\left\|x_{n}-x^{*}\right\|^{2}+\left\langle\gamma f\left(x^{*}\right)-\mu A x^{*}, x_{n}-x^{*}\right\rangle\right) .
\end{aligned}
$$

This implies that

$$
\left\|x_{n}-x^{*}\right\|^{2} \leq \frac{\left\langle\gamma f\left(x^{*}\right)-\mu A x^{*}, x_{n}-x^{*}\right\rangle}{\tau-\gamma \alpha} .
$$

In particular,

$$
\left\|x_{n_{j}}-x^{*}\right\|^{2} \leq \frac{\left\langle\gamma f\left(x^{*}\right)-\mu A x^{*}, x_{n_{j}}-x^{*}\right\rangle}{\tau-\gamma \alpha} .
$$

Since $x_{n_{j}} \rightarrow x^{*}$, it follows from (3.31) that $x_{n_{j}} \rightarrow x^{*}$ as $j \rightarrow \infty$. Next, we show that $x^{*}$ solves the variational inequality (3.4).

By the iterative algorithm (3.5), we have

$$
x_{n}=\alpha_{n} \gamma f\left(x_{n}\right)+\left(I-\mu \alpha_{n} A\right) y_{n}=\alpha_{n} \gamma f\left(x_{n}\right)+\left(I-\mu \alpha_{n} A\right) V_{n} x_{n} .
$$


Therefore, we have

$$
\mu \alpha_{n} A x_{n}-\alpha_{n} \gamma f\left(x_{n}\right)=\mu \alpha_{n} A x_{n}-x_{n}+\left(I-\mu \alpha_{n} A\right) V_{n} x_{n}
$$

that is,

$$
(\mu A-\gamma f) x_{n}=-\frac{1}{\alpha_{n}}\left(\left(I-V_{n}\right) x_{n}-\mu \alpha_{n}\left(A x_{n}-A V_{n}\right) x_{n}\right)
$$

Hence, for $p \in \Omega$,

$$
\begin{aligned}
\left\langle(\mu A-\gamma f) x_{n}, x_{n}-p\right\rangle & =-\frac{1}{\alpha_{n}}\left\langle\left(I-V_{n}\right) x_{n}-\mu \alpha_{n}\left(A x_{n}-A V_{n} x_{n}\right), x_{n}-p\right\rangle \\
& =-\frac{1}{\alpha_{n}}\left\langle\left(I-V_{n}\right) x_{n}-\left(I-V_{n}\right) p, x_{n}-p\right\rangle+\mu\left\langle A x_{n}-A V_{n} x_{n}, x_{n}-p\right\rangle \\
& \leq \mu\left\langle A x_{n}-A V_{n} x_{n}, x_{n}-p\right\rangle .
\end{aligned}
$$

Since $I-V_{n}$ is monotone (i.e., $\left\langle x-y,\left(I-V_{n}\right) x-\left(I-V_{n}\right) y\right\rangle \geq 0$, for all $\left.x, y \in H\right)$. This is due to the nonexpansivity of $V_{n}$.

Now replacing $n$ in (3.35) with $n_{j}$ and letting $j \rightarrow \infty$, we obtain

$$
\left\langle(\mu A-\gamma f) x^{*}, x^{*}-p\right\rangle=\lim _{j \rightarrow \infty}\left\langle(\mu A-\gamma f) x_{n_{j}}, x_{n_{j}}-p\right\rangle \leq \lim _{j \rightarrow \infty} \mu\left\langle A x_{n_{j}}-A V_{n} x_{n_{j}}, x_{n_{j}}-p\right\rangle=0 .
$$

That is, $x^{*} \in \Omega$ is a solution of (3.4). To show that the sequence $\left\{x_{n}\right\}$ converges strongly to $x^{*}$, we assume that $x_{n_{k}} \rightarrow \widehat{x}$. By the same processing as the proof above, we derive $\widehat{x} \in \Omega$. Moreover, it follows from the inequality (3.36) that

$$
\left\langle(\mu A-\gamma f) x^{*}, x^{*}-\widehat{x}\right\rangle \leq 0 .
$$

Interchanging $x^{*}$ and $\widehat{x}$, we get

$$
\left\langle(\mu A-\gamma f) \widehat{x}, \widehat{x}-x^{*}\right\rangle \leq 0
$$

By Lemma 2.5, adding up (3.37) and (3.38) yields

$$
(\mu \eta-\gamma \alpha)\left\|x^{*}-\widehat{x}\right\|^{2} \leq\left\langle(\mu A-\gamma f) x^{*}-(\mu A-\gamma f) \hat{x}, x^{*}-\widehat{x}\right\rangle \leq 0 .
$$

Hence $x^{*}=\widehat{x}$ and, therefore, $x_{n} \rightarrow x^{*}$ as $n \rightarrow \infty$,

$$
\left\langle(I-\mu A+\gamma f) x^{*}-x^{*}, x^{*}-p\right\rangle \geq 0, \quad \forall p \in \Omega .
$$


This is equivalent to the fixed point equation:

$$
P_{\Omega}(I-\mu A+\gamma f) x^{*}=x^{*} .
$$

Theorem 3.2. Let $C$ be a nonempty closed convex subset of a real Hilbert space $H$ and $F$ a bifunction from $C \times C$ to $\mathbb{R}$ satisfying (A1)-(A4). Let $S_{i}: C \rightarrow C$ be a family $\kappa_{i}$-strict pseudocontractions for some $0 \leq \kappa_{i}<1$. Assume the set $\Omega=\cap_{i=1}^{\infty} F\left(S_{i}\right) \cap E P(F) \neq \emptyset$. Let $f$ be a contraction of $H$ into itself with $\alpha \in(0,1)$ and let $A$ be a L-Lipschitzian continuous operator and $\eta$-strongly monotone with $L>0, \eta>0,0<\mu<2 \eta / L^{2}$, and $0<\gamma<\mu\left(\eta-\left(\mu L^{2} / 2\right)\right) / \alpha=\tau / \alpha$. For every $n \in \mathbb{N}$, let $W_{n}$ be the mapping generated by $S_{i}^{\prime}$ and $0<t_{i} \leq b<1$. Given $x_{1} \in H$, let $\left\{x_{n}\right\}$ and $\left\{u_{n}\right\}$ be sequences generated by the following algorithm:

$$
\begin{aligned}
u_{n} & =T_{r_{n}} x_{n}, \\
y_{n} & =\beta_{n} x_{n}+\left(1-\beta_{n}\right) W_{n} u_{n}, \\
x_{n+1} & =\alpha_{n} \gamma f\left(x_{n}\right)+\left(I-\mu \alpha_{n} A\right) y_{n} .
\end{aligned}
$$

If $\left\{\alpha_{n}\right\},\left\{\beta_{n}\right\}$ and $\left\{r_{n}\right\}$ satisfy the following conditions:

(i) $\left\{\alpha_{n}\right\} \subset(0,1), \lim _{n \rightarrow \infty} \alpha_{n}=0$ and $\sum_{n=1}^{\infty} \alpha_{n}=\infty$;

(ii) $0<\liminf _{n \rightarrow \infty} \beta_{n} \leq \limsup \sup _{n \rightarrow \infty} \beta_{n}<1$;

(iii) $\left\{r_{n}\right\} \subset(0, \infty), \liminf _{n \rightarrow \infty} r_{n}>0$ and $\lim _{n \rightarrow \infty}\left|r_{n+1}-r_{n}\right|=0$.

Then, $\left\{x_{n}\right\}$ converges strongly to $x^{*} \in \Omega$, which solves the variational inequality (3.4).

Proof. The proof is divided into several steps.

Step 1. Show first that $\left\{x_{n}\right\}$ is bounded.

Taking any $p \in \Omega$, we have

$$
\begin{aligned}
\left\|x_{n+1}-p\right\| & =\left\|\alpha_{n}\left(\gamma f\left(x_{n}\right)-\mu A p\right)+\left(I-\mu \alpha_{n} A\right) y_{n}-\left(I-\mu \alpha_{n} A\right) p\right\| \\
& \leq \alpha_{n}\left(\left\|\gamma f\left(x_{n}\right)-\gamma f(p)\right\|+\|\gamma f(p)-\mu A p\|\right)+\left(1-\alpha_{n} \tau\right)\left\|y_{n}-p\right\| \\
& \leq \alpha_{n} \alpha \gamma\left\|x_{n}-p\right\|+\alpha_{n}\|\gamma f(p)-\mu A p\|+\left(1-\alpha_{n} \tau\right)\left\|y_{n}-p\right\| \\
& =\left(1-\alpha_{n}(\tau-\alpha \gamma)\right)\left\|x_{n}-p\right\|+\alpha_{n}(\tau-\alpha \gamma) \frac{\|\gamma f(p)-\mu A p\|}{\tau-\alpha \gamma} \\
& \leq \max \left\{\left\|x_{n}-p\right\|, \frac{\|\gamma f(p)-\mu A p\|}{\tau-\alpha \gamma}\right\} .
\end{aligned}
$$

By induction, we obtain $\left.\left\|x_{n}-p\right\| \leq \max \left\{\left\|x_{1}-p\right\|, \| \gamma f(p)-\mu A p\right) \| /(\tau-\alpha \gamma)\right\}, n \geq 1$. Hence, $\left\{x_{n}\right\}$ is bounded, so are $\left\{u_{n}\right\}$ and $\left\{y_{n}\right\}$. It follows from the Lipschitz continuity of $A$ that $\left\{A x_{n}\right\}$ and $\left\{A u_{n}\right\}$ are also bounded. From the nonexpansivity of $f$ and $W_{n}$, it follows that $\left\{f\left(x_{n}\right)\right\}$ and $\left\{W_{n} x_{n}\right\}$ are also bounded. 
Step 2. Show that

$$
\left\|x_{n+1}-x_{n}\right\| \longrightarrow 0
$$

Observe that

$$
\begin{aligned}
\left\|u_{n+1}-u_{n}\right\| & =\left\|T_{r_{n+1}} x_{n+1}-T_{r_{n}} x_{n}\right\| \\
& \leq\left\|T_{r_{n+1}} x_{n+1}-T_{r_{n+1}} x_{n}\right\|+\left\|T_{r_{n+1}} x_{n}-T_{r_{n}} x_{n}\right\| \\
& \leq\left\|x_{n+1}-x_{n}\right\|+\left\|T_{r_{n+1}} x_{n}-T_{r_{n}} x_{n}\right\|,
\end{aligned}
$$

and from (2.5), we have

$$
\begin{aligned}
\left\|W_{n+1} u_{n}-W_{n} u_{n}\right\| & =\left\|t_{1} S_{1}^{\prime} U_{n+1,2} u_{n}-t_{1} S_{1}^{\prime} U_{n, 2} u_{n}\right\| \\
& \leq t_{1}\left\|U_{n+1,2} u_{n}-U_{n, 2} u_{n}\right\| \\
& =t_{1}\left\|t_{2} S_{2}^{\prime} U_{n+1,3} u_{n}-t_{2} S_{2}^{\prime} U_{n, 3} u_{n}\right\| \\
& \leq t_{1} t_{2}\left\|U_{n+1,3} u_{n}-U_{n, 3} u_{n}\right\| \\
& \leq \cdots \\
& \leq \prod_{i=1}^{n} t_{i}\left\|U_{n+1, n+1} u_{n}-U_{n, n+1} u_{n}\right\| \\
& \leq M_{1} \prod_{i=1}^{n} t_{i}
\end{aligned}
$$

where $M_{1}=\sup _{n}\left\{\left\|U_{n+1, n+1} u_{n}-U_{n, n+1} u_{n}\right\|\right\}$.

Suppose $x_{n+1}=\beta_{n} x_{n}+\left(1-\beta_{n}\right) z_{n}$, then $z_{n}=\left(x_{n+1}-\beta_{n} x_{n}\right) /\left(1-\beta_{n}\right)=\left(\alpha_{n} \gamma f\left(x_{n}\right)+(I-\right.$ $\left.\left.\mu \alpha_{n} A\right) y_{n}-\beta_{n} x_{n}\right) /\left(1-\beta_{n}\right)$.

Hence, we have

$$
\begin{aligned}
z_{n+1}-z_{n}= & \frac{\alpha_{n+1} \gamma f\left(x_{n+1}\right)+\left(I-\mu \alpha_{n+1} A\right) y_{n+1}-\beta_{n+1} x_{n+1}}{1-\beta_{n+1}}-\frac{\alpha_{n} \gamma f\left(x_{n}\right)+\left(I-\mu \alpha_{n} A\right) y_{n}-\beta_{n} x_{n}}{1-\beta_{n}} \\
= & \frac{\alpha_{n+1}\left(\gamma f\left(x_{n+1}\right)-\mu A y_{n+1}\right)}{1-\beta_{n+1}}+\frac{y_{n+1}-\beta_{n+1} x_{n+1}}{1-\beta_{n+1}}-\frac{\alpha_{n}\left(\gamma f\left(x_{n}\right)-\mu A y_{n}\right)}{1-\beta_{n}}-\frac{y_{n}-\beta_{n} x_{n}}{1-\beta_{n}} \\
= & \frac{\alpha_{n+1}\left(\gamma f\left(x_{n+1}\right)-\mu A y_{n+1}\right)}{1-\beta_{n+1}}+\frac{\beta_{n+1} x_{n+1}+\left(1-\beta_{n+1}\right) W_{n+1} u_{n+1}-\beta_{n+1} x_{n+1}}{1-\beta_{n+1}} \\
& -\frac{\alpha_{n}\left(\gamma f\left(x_{n}\right)-\mu A y_{n}\right)}{1-\beta_{n}}-\frac{\beta_{n} x_{n}+\left(1-\beta_{n}\right) W_{n} u_{n}-\beta_{n} x_{n}}{1-\beta_{n}} \\
\leq & \frac{\alpha_{n+1}\left(\gamma f\left(x_{n+1}\right)-\mu A y_{n+1}\right)}{1-\beta_{n+1}}-\frac{\alpha_{n}\left(\gamma f\left(x_{n}\right)-\mu A y_{n}\right)}{1-\beta_{n}}+W_{n+1} u_{n+1}-W_{n} u_{n} .
\end{aligned}
$$


It follows from (3.45), (3.46), and the above result that

$$
\begin{aligned}
& \left\|z_{n+1}-z_{n}\right\| \\
& \quad \leq \frac{\alpha_{n+1}}{1-\beta_{n+1}}\left(\left\|\gamma f\left(x_{n+1}\right)\right\|+\left\|\mu A y_{n+1}\right\|\right)+\frac{\alpha_{n}}{1-\beta_{n}}\left(\left\|\gamma f\left(x_{n}\right)\right\|+\left\|\mu A y_{n}\right\|\right)+\left\|W_{n+1} u_{n+1}-W_{n} u_{n}\right\| \\
& \quad \leq\left(\frac{\alpha_{n+1}}{1-\beta_{n+1}}+\frac{\alpha_{n}}{1-\beta_{n}}\right) M_{2}+\left\|W_{n+1} u_{n+1}-W_{n+1} u_{n}\right\|+\left\|W_{n+1} u_{n}-W_{n} u_{n}\right\| \\
& \quad \leq\left(\frac{\alpha_{n+1}}{1-\beta_{n+1}}+\frac{\alpha_{n}}{1-\beta_{n}}\right) M_{2}+\left\|u_{n+1}-u_{n}\right\|+\left\|W_{n+1} u_{n}-W_{n} u_{n}\right\| \\
& \quad \leq\left\|x_{n+1}-x_{n}\right\|+\left\|T_{r_{n+1}} x_{n}-T_{r_{n}} x_{n}\right\|+\left(\frac{\alpha_{n+1}}{1-\beta_{n+1}}+\frac{\alpha_{n}}{1-\beta_{n}}\right) M_{2}+M_{1} \prod_{i=1}^{n} t_{i}
\end{aligned}
$$

where $M_{2}=\sup _{n}\left\{\left\|\gamma f\left(x_{n}\right)\right\|+\left\|\mu A y_{n}\right\|\right\}$. Hence, we get

$$
\left\|z_{n+1}-z_{n}\right\|-\left\|x_{n+1}-x_{n}\right\| \leq\left\|T_{r_{n+1}} x_{n}-T_{r_{n}} x_{n}\right\|+\left(\frac{\alpha_{n+1}}{1-\beta_{n+1}}+\frac{\alpha_{n}}{1-\beta_{n}}\right) M_{2}+M_{1} \prod_{i=1}^{n} t_{i} .
$$

From condition (i), (iii), $0<t_{n} \leq b<1$, and Lemma 2.12, we obtain

$$
\limsup _{n \rightarrow \infty}\left(\left\|z_{n+1}-z_{n}\right\|-\left\|x_{n+1}-x_{n}\right\|\right) \leq 0
$$

By Lemma 2.11,we have $\lim _{n \rightarrow \infty}\left\|z_{n}-x_{n}\right\|=0$. Thus,

$$
\lim _{n \rightarrow \infty}\left\|x_{n+1}-x_{n}\right\|=\lim _{n \rightarrow \infty}\left(1-\beta_{n}\right)\left\|z_{n}-x_{n}\right\|=0 .
$$

By Lemma 2.12, (3.45) and (3.44), we obtain

$$
\left\|u_{n+1}-u_{n}\right\| \longrightarrow 0
$$

Step 3. Show that

$$
\left\|x_{n}-W x_{n}\right\| \longrightarrow 0
$$

Observe that

$$
\begin{gathered}
\left\|x_{n}-W_{n} x_{n}\right\| \leq\left\|x_{n}-W_{n} u_{n}\right\|+\left\|W_{n} u_{n}-W_{n} x_{n}\right\| \leq\left\|x_{n}-W_{n} u_{n}\right\|+\left\|u_{n}-x_{n}\right\|, \\
\left\|x_{n}-W_{n} u_{n}\right\| \leq\left\|x_{n}-x_{n+1}\right\|+\left\|x_{n+1}-y_{n}\right\|+\left\|y_{n}-W_{n} u_{n}\right\|=\left\|x_{n}-x_{n+1}\right\| \\
+\left\|x_{n+1}-y_{n}\right\|+\beta_{n}\left(\left\|u_{n}-x_{n}\right\|+\left\|x_{n}-W_{n} u_{n}\right\|\right) .
\end{gathered}
$$


From condition (i) and (3.5), we can obtain

$$
\begin{aligned}
\left(1-\beta_{n}\right)\left\|x_{n}-W_{n} u_{n}\right\| & \leq\left\|x_{n}-x_{n+1}\right\|+\left\|x_{n+1}-y_{n}\right\|+\beta_{n}\left\|u_{n}-x_{n}\right\| \\
& \leq\left\|x_{n}-x_{n+1}\right\|+\alpha_{n}\left\|\gamma f\left(x_{n}\right)-\mu A y_{n}\right\|+\beta_{n}\left\|u_{n}-x_{n}\right\| .
\end{aligned}
$$

By Lemma 2.10, we get

$$
\left\|u_{n}-p\right\|^{2}=\left\|T_{r_{n}} x_{n}-T_{r_{n}} p\right\|^{2} \leq\left\langle T_{r_{n}} x_{n}-T_{r_{n}} p, x_{n}-p\right\rangle=\frac{1}{2}\left(\left\|u_{n}-p\right\|^{2}+\left\|x_{n}-p\right\|^{2}+\left\|x_{n}-u_{n}\right\|^{2}\right) .
$$

This implies that

$$
\left\|u_{n}-p\right\|^{2} \leq\left\|x_{n}-p\right\|^{2}-\left\|x_{n}-u_{n}\right\|^{2} .
$$

By nonexpansivity of $W_{n}$, we have

$$
\left\|y_{n}-p\right\|^{2} \leq \beta_{n}\left\|x_{n}-p\right\|^{2}+\left(1-\beta_{n}\right)\left\|u_{n}-p\right\|^{2} \leq\left\|x_{n}-p\right\|^{2}-\left(1-\beta_{n}\right)\left\|x_{n}-u_{n}\right\|^{2} .
$$

It follows from (3.42) that

$$
\begin{aligned}
\left\|x_{n+1}-p\right\|^{2} & =\left\|\alpha_{n}\left(\gamma f\left(x_{n}\right)-p\right)+\left(I-\mu \alpha_{n} A\right) y_{n}-\left(I-\mu \alpha_{n} A\right) p+\alpha_{n}(p-\mu A p)\right\|^{2} \\
& \leq \alpha_{n}\left\|\gamma f\left(x_{n}\right)-p\right\|^{2}+\left(1-\alpha_{n} \tau\right)\left\|y_{n}-p\right\|^{2}+\alpha_{n}\|p-\mu A p\|^{2} \\
& \leq \alpha_{n}\left\|\gamma f\left(x_{n}\right)-p\right\|^{2}+\left(1-\alpha_{n} \tau\right)\left(\left\|x_{n}-p\right\|^{2}-\left(1-\beta_{n}\right)\left\|x_{n}-u_{n}\right\|^{2}\right)+\alpha_{n}\|p-\mu A p\|^{2} \\
& \leq \alpha_{n}\left\|\gamma f\left(x_{n}\right)-p\right\|^{2}+\left\|x_{n}-p\right\|^{2}-\left(1-\beta_{n}\right)\left\|x_{n}-u_{n}\right\|^{2}+\alpha_{n}\|p-\mu A p\|^{2} .
\end{aligned}
$$

This implies that

$$
\begin{aligned}
\left(1-\beta_{n}\right)\left\|x_{n}-u_{n}\right\|^{2} & \leq \alpha_{n}\left(\left\|\gamma f\left(x_{n}\right)-p\right\|^{2}+\|p-\mu A p\|^{2}\right)+\left\|x_{n}-p\right\|^{2}-\left\|x_{n+1}-p\right\|^{2} \\
& \leq \alpha_{n}\left(\left\|\gamma f\left(x_{n}\right)-p\right\|^{2}+\|p-\mu A p\|^{2}\right)+\left(\left\|x_{n}-p\right\|+\left\|x_{n+1}-p\right\|\right)\left\|x_{n+1}-x_{n}\right\| .
\end{aligned}
$$

From condition (i), (ii), and (3.44), we have

$$
\left\|x_{n}-u_{n}\right\| \longrightarrow 0
$$

Further we have $\left\|x_{n}-W_{n} u_{n}\right\| \rightarrow 0$. Thus we get

$$
\left\|x_{n}-W_{n} x_{n}\right\| \longrightarrow 0
$$


On the other hand, we have

$$
\left\|x_{n}-W x_{n}\right\| \leq\left\|x_{n}-W_{n} x_{n}\right\|+\left\|W_{n} x_{n}-W x_{n}\right\| \leq\left\|x_{n}-W_{n} x_{n}\right\|+\sup _{x_{n} \in C}\left\|W_{n} x_{n}-W x_{n}\right\| .
$$

Combining (3.62), the last inequality, and Lemma 2.7, we obtain (3.53).

Step 4. Show that

$$
\limsup _{n \rightarrow \infty}\left\langle(\gamma f-\mu A) x^{*}, x_{n}-x^{*}\right\rangle \leq 0,
$$

where $x^{*}=P_{\Omega}(I-\mu A+\gamma f) x^{*}$ is a unique solution of the variational inequality (3.4). Indeed, take a subsequence $\left\{x_{n_{j}}\right\}$ of $\left\{x_{n}\right\}$ such that

$$
\limsup _{n \rightarrow \infty}\left\langle(\gamma f-\mu A) x^{*}, x_{n}-x^{*}\right\rangle=\lim _{j \rightarrow \infty}\left\langle(\gamma f-\mu A) x^{*}, x_{n_{j}}-x^{*}\right\rangle .
$$

Since $\left\{x_{n_{j}}\right\}$ is bounded, there exists a subsequence $\left\{x_{n_{j_{k}}}\right\}$ of $\left\{x_{n_{j}}\right\}$ which converges weakly to $q$. Without loss of generality, we can assume $x_{n_{j}} \rightarrow q$. From (3.53), we obtain $W x_{n_{j}} \rightarrow q$.

By the same argument as in the proof of Theorem 3.1, we have $q \in \Omega$. Since $x^{*}=$ $P_{\Omega}(I-\mu A+\gamma f) x^{*}$, it follows that

$$
\limsup _{n \rightarrow \infty}\left\langle(\gamma f-\mu A) x^{*}, x_{n}-x^{*}\right\rangle=\lim _{j \rightarrow \infty}\left\langle(\gamma f-\mu A) x^{*}, x_{n_{j}}-x^{*}\right\rangle=\left\langle(\gamma f-\mu A) x^{*}, q-x^{*}\right\rangle \leq 0 .
$$

Step 5. Show that

$$
x_{n} \longrightarrow x^{*} \text {. }
$$

Since

$$
\begin{aligned}
\left\langle(\gamma f-\mu A) x^{*}, x_{n+1}-x^{*}\right\rangle & =\left\langle(\gamma f-\mu A) x^{*}, x_{n+1}-x_{n}\right\rangle+\left\langle(\gamma f-\mu A) x^{*}, x_{n}-x^{*}\right\rangle \\
& \leq\left\|(\gamma f-\mu A) x^{*}\right\|\left\|x_{n+1}-x_{n}\right\|+\left\langle(\gamma f-\mu A) x^{*}, x_{n}-x^{*}\right\rangle .
\end{aligned}
$$

It follows from (3.44) and (3.66) that

$$
\begin{aligned}
& \limsup _{n \rightarrow \infty}\left\langle(\gamma f-\mu A) x^{*}, x_{n+1}-x^{*}\right\rangle \leq 0 . \\
& \left\|x_{n+1}-x^{*}\right\|^{2} \\
& =\left\|\alpha_{n} \gamma f\left(x_{n}\right)+\left(I-\mu \alpha_{n} A\right) y_{n}-x^{*}\right\|^{2} \\
& =\left\|\left(I-\mu \alpha_{n} A\right) y_{n}-\left(I-\mu \alpha_{n} A\right) x^{*}+\alpha_{n}\left(\gamma f\left(x_{n}\right)-\mu A x^{*}\right)\right\|^{2}
\end{aligned}
$$




$$
\begin{aligned}
& \leq\left\|\left(I-\mu \alpha_{n} A\right) y_{n}-\left(I-\mu \alpha_{n} A\right) x^{*}\right\|^{2}+2 \alpha_{n}\left\langle\gamma f\left(x_{n}\right)-\mu A x^{*}, x_{n+1}-x^{*}\right\rangle \\
& \leq\left(1-\alpha_{n} \tau\right)^{2}\left\|y_{n}-x^{*}\right\|^{2}+2 \alpha_{n}\left\langle\gamma f\left(x_{n}\right)-\gamma f\left(x^{*}\right), x_{n+1}-x^{*}\right\rangle+2 \alpha_{n}\left\langle(\gamma f-\mu A) x^{*}, x_{n+1}-x^{*}\right\rangle \\
& \leq\left(1-\alpha_{n} \tau\right)^{2}\left\|x_{n}-x^{*}\right\|^{2}+\alpha_{n} \alpha \gamma\left(\left\|x_{n}-x^{*}\right\|^{2}+\left\|x_{n+1}-x^{*}\right\|^{2}\right)+2 \alpha_{n}\left\langle(\gamma f-\mu A) x^{*}, x_{n+1}-x^{*}\right\rangle .
\end{aligned}
$$

This implies that

$$
\begin{aligned}
\| x_{n+1} & -x^{*} \|^{2} \\
& \leq \frac{\left(1-\alpha_{n} \tau\right)^{2}+\alpha_{n} \alpha \gamma}{1-\alpha_{n} \alpha \gamma}\left\|x_{n}-x^{*}\right\|^{2}+\frac{2 \alpha_{n}}{1-\alpha_{n} \alpha \gamma}\left\langle(\gamma f-\mu A) x^{*}, x_{n+1}-x^{*}\right\rangle \\
& \leq\left(1-\frac{2 \alpha_{n}(\tau-\alpha \gamma)}{1-\alpha_{n} \alpha \gamma}\right)\left\|x_{n}-x^{*}\right\|^{2}+\frac{2 \alpha_{n}}{1-\alpha_{n} \alpha \gamma}\left\langle(\gamma f-\mu A) x^{*}, x_{n+1}-x^{*}\right\rangle+\frac{\left(\alpha_{n} \tau\right)^{2}}{1-\alpha_{n} \alpha \gamma} M_{3},
\end{aligned}
$$

where $M_{3}=\sup _{n}\left\|x_{n}-x^{*}\right\|^{2}, n \geq 1$. It is easily to see that $\gamma_{n}=2 \alpha_{n}(\tau-\alpha \gamma) /\left(1-\alpha_{n} \alpha \gamma\right)$. Hence, by Lemma 2.2, the sequence $\left\{x_{n}\right\}$ converges strongly to $x^{*}$.

Remark 3.3. If $F \equiv 0$, then Theorem 3.2 reduces to Theorem 3.1 of Wang [10].

\section{Acknowledgments}

The authors would like to thank the referee for valuable suggestions to improve the manuscript NSFC Tianyuan Youth Foundation of Mathematics of China (no. 11126136), and the Fundamental Research Funds for the Central Universities (GRANT: ZXH2011C002).

\section{References}

[1] F. E. Browder and W. V. Petryshyn, "Construction of fixed points of nonlinear mappings in Hilbert space," Journal of Mathematical Analysis and Applications, vol. 20, pp. 197-228, 1967.

[2] Y. Liu, "A general iterative method for equilibrium problems and strict pseudo-contractions in Hilbert spaces," Nonlinear Analysis: Theory, Methods \& Applications, vol. 71, no. 10, pp. 4852-4861, 2009.

[3] G. Marino and H.-K. Xu, "Weak and strong convergence theorems for strict pseudo-contractions in Hilbert spaces," Journal of Mathematical Analysis and Applications, vol. 329, no. 1, pp. 336-346, 2007.

[4] S. Takahashi and W. Takahashi, "Strong convergence theorem for a generalized equilibrium problem and a nonexpansive mapping in a Hilbert space," Nonlinear Analysis: Theory, Methods E Applications, vol. 69, no. 3, pp. 1025-1033, 2008.

[5] P. L. Combettes and S. A. Hirstoaga, "Equilibrium programming in Hilbert spaces," Journal of Nonlinear and Convex Analysis, vol. 6, no. 1, pp. 117-136, 2005.

[6] W. R. Mann, "Mean value methods in iteration," Proceedings of the American Mathematical Society, vol. 4, pp. 506-510, 1953.

[7] K. Shimoji and W. Takahashi, "Strong convergence to common fixed points of infinite nonexpansive mappings and applications," Taiwanese Journal of Mathematics, vol. 5, no. 2, pp. 387-404, 2001.

[8] G. L. Acedo and H.-K. Xu, "Iterative methods for strict pseudo-contractions in Hilbert spaces," Nonlinear Analysis: Theory, Methods E Applications, vol. 67, no. 7, pp. 2258-2271, 2007. 
[9] M. Tian, "A general iterative algorithm for nonexpansive mappings in Hilbert spaces," Nonlinear Analysis: Theory, Methods \& Applications, vol. 73, no. 3, pp. 689-694, 2010.

[10] S. Wang, "A general iterative method for obtaining an infinite family of strictly pseudo-contractive mappings in Hilbert spaces," Applied Mathematics Letters, vol. 24, no. 6, pp. 901-907, 2011.

[11] H. K. Xu, "An iterative approach to quadratic optimization," Journal of Optimization Theory and Applications, vol. 116, no. 3, pp. 659-678, 2003.

[12] S.-S. Chang, H. W. Joseph Lee, and C. K. Chan, "A new method for solving equilibrium problem fixed point problem and variational inequality problem with application to optimization," Nonlinear Analysis: Theory, Methods \& Applications, vol. 70, no. 9, pp. 3307-3319, 2009.

[13] E. Blum and W. Oettli, "From optimization and variational inequalities to equilibrium problems," The Mathematics Student, vol. 63, no. 1-4, pp. 123-145, 1994.

[14] T. Suzuki, "Strong convergence of Krasnoselskii and Mann's type sequences for one-parameter nonexpansive semigroups without Bochner integrals," Journal of Mathematical Analysis and Applications, vol. 305, no. 1, pp. 227-239, 2005. 


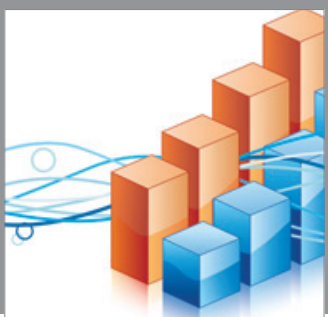

Advances in

Operations Research

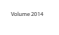

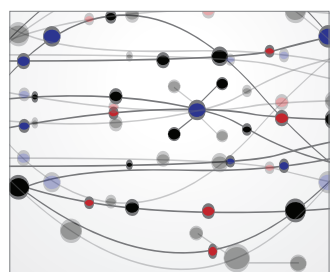

\section{The Scientific} World Journal
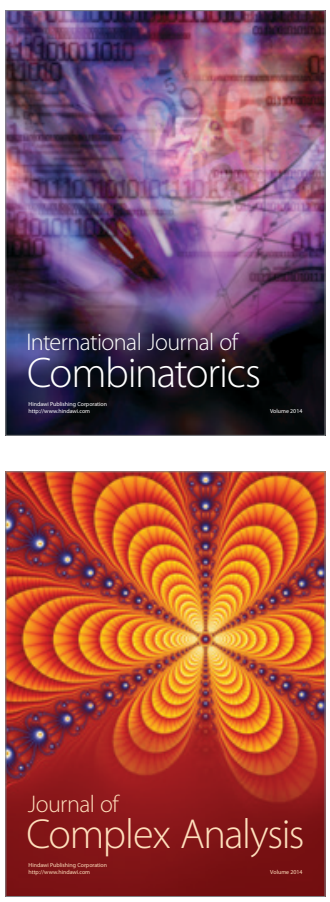

International Journal of

Mathematics and

Mathematical

Sciences
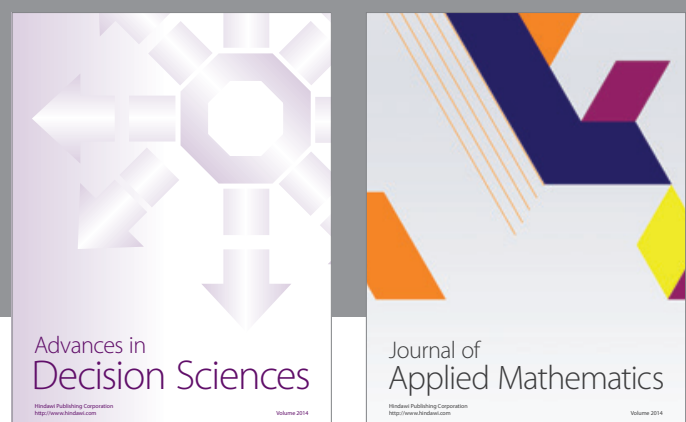

Journal of

Applied Mathematics
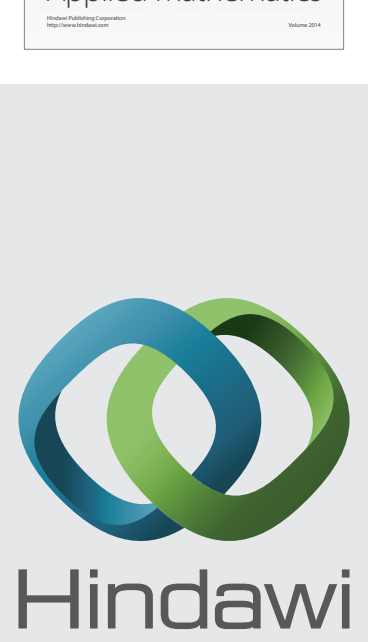

Submit your manuscripts at http://www.hindawi.com
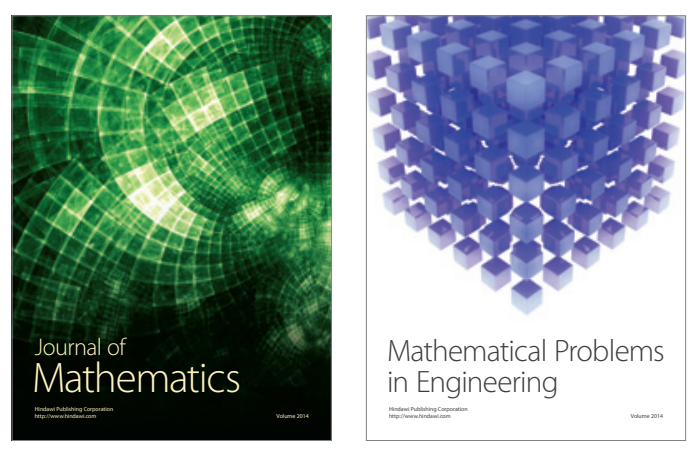

Mathematical Problems in Engineering
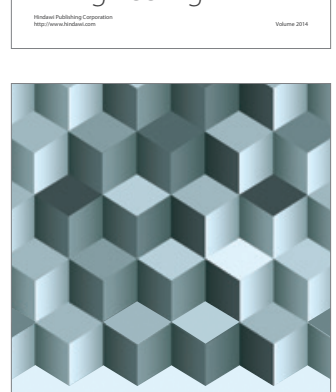

Journal of

Function Spaces
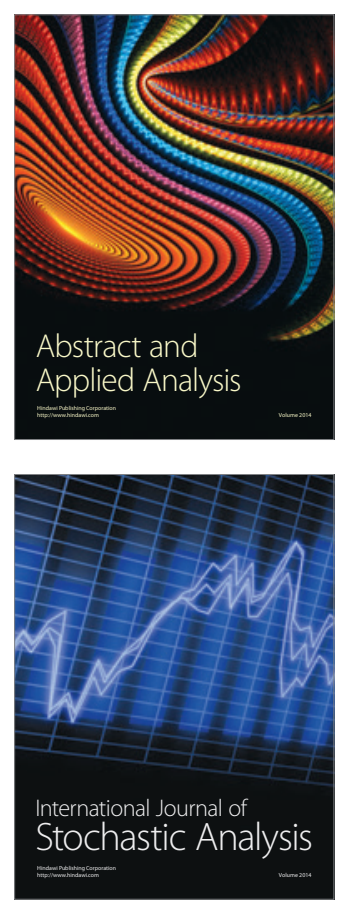

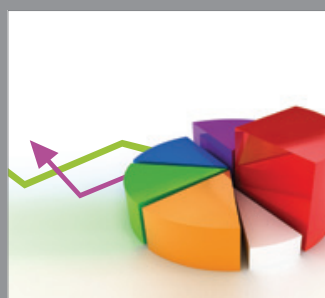

ournal of

Probability and Statistics

Promensencen
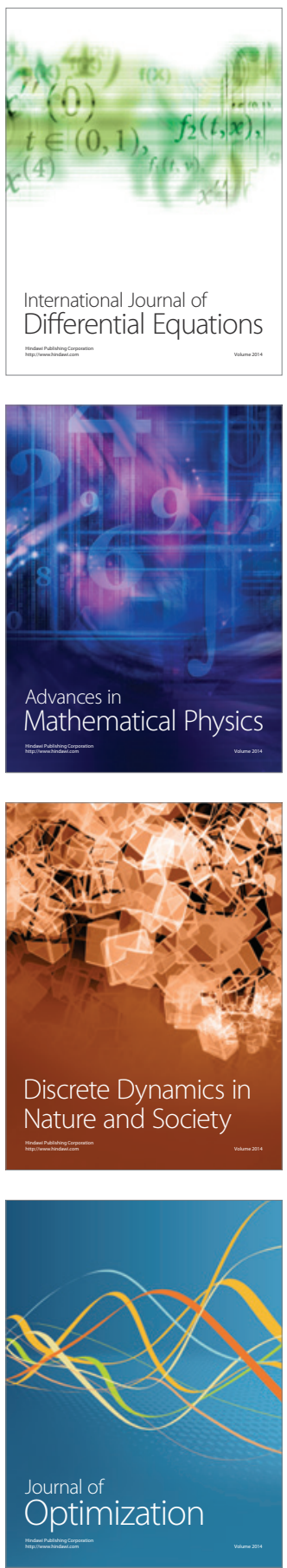